\title{
An Exploration of Taste-Emotion Mappings from the Perspective of Food Design Practitioners
}

\author{
Tom Gayler \\ Lancaster University \\ LA1 4WA \\ United Kingdom \\ t.gayler@lancaster.ac.uk
}

\author{
Corina Sas \\ Lancaster University \\ LA1 4WA \\ United Kingdom \\ c.sas@lancaster.ac.uk
}

\begin{abstract}
This paper explores taste-emotion mappings and how they may inform the design of user experience in HCI. We report interviews with 7 food industry professionals and discuss the findings against laboratory-based psychology studies. While the sweet-positive affect and bitter-negative affect mappings were confirmed, those for sour, salty and umami tastes were challenged. Our outcomes highlight a more nuanced understanding of taste-emotion mappings, the influence of taste intensity and the importance of narrative and temporality when designing taste experience in naturalistic settings.
\end{abstract}

\section{CCS CONCEPTS}

Human-centered computing $\rightarrow$ Human computer interaction $(\mathrm{HCI}) \rightarrow$ Interaction paradigms

\section{KEYWORDS}

3D Printed Food, Taste, Emotion, Affective Computing

\section{ACM Reference format:}

T. Gayler, C. Sas. 2017. SIG Proceedings Paper in word Format. In Proceedings of 2nd ACM SIGCHI International Workshop on Multisensory Approaches to Human-Food Interaction, Glasgow, UK, 13 ${ }^{\text {th }}$ Novemeber 2017 (MHFI'17), 4 pages.

DOI: $10.1145 / 3141788.3141793$

\section{INTRODUCTION}

Food is an interesting design material which has received increasing HCI interest. Whilst a few prior studies have started exploring interactive systems around food, there has been little work on food as an interactive medium. With the rise of technologies such as 3D food printers there is now a real possibility of developing 'edible interfaces' [36], challenging

Permission to make digital or hard copies of all or part of this work for personal or classroom use is granted without fee provided that copies are not made or distributed for profit or commercial advantage and that copies bear this notice and the full citation on the first page. Copyrights for components of this work owned by others than ACM must be honored. Abstracting with credit is permitted. To copy otherwise, or republish, to post on servers or to redistribute to lists, requires prior specific permission and/or a fee. Request permissions from Permissions@acm.org.

MHFI'17, November 13, 2017, Glasgow, United Kingdom (C) 2017 Association for Computing Machinery.

ACM ISBN 978-1-4503-5556-8/17/11...\$15.00

https://doi.org/10.1145/3141788.3141793 designers to understand how food can be leveraged to create novel and meaningful user experiences. A promising possibility for edible interfaces arises from the connection of taste and emotion. In lab-based studies there is a weight of evidence showing mappings between basic tastes and specific affective responses [3,14,23,28,39]. We know little, however, of how such mappings apply to food experience in everyday, ecologically valid contexts.

In this paper, we report on interviews with 7 experts in food design to explore their understanding of how tastes facilitate various affective responses, and of specific foodstuff illustrating such mappings. For this, we are drawing from previous work on the psychology of taste and emotions, as well as theories of embodiment, and focused on the following research questions:

- Is sweet taste predominantly mapped to positive emotions as suggested by psychology of taste?

- Are sour, bitter and salty tastes predominantly mapped to negative emotions?

- How does the intensity of the taste link to emotional responses?

\section{BACKGROUND RESEARCH}

Much previous work on tastes and emotions has shown their relationship, both in terms of mappings of emotional responses to tastes $[3,23,28,39]$ and as tastes' influence on the perception of affective stimuli $[8,25]$. Most of these existing studies rely on pure tastants (that only trigger taste receptors) as their stimulus, and occur in controlled, laboratory environments. Thus, their findings tend to lack the ecologically validity required to support their use in an applied field such as HCI.

Sweet taste has been shown to map predominantly to positive emotions $[23,35,37,39]$ and influence mood and moral decision making through enhancing positive, and limiting negative responses to affective stimulus [8,11]. Negative affective response has been linked to bitter [12,14,17,28,35], sour $[28,37,39]$ and salty tastes $[23,28,35,37,39]$. Disgust specifically has been mapped to bitter [3,8,38], sour and salty tastes $[3,26]$. Bitter taste specifically has been used to explore the influence of embodied cognition $[6,8]$.

Among the few studies conducted under ecologically valid conditions is Noel and Dando's [20] exploration of the relationships between taste and emotion following hockey matches. At a series of matches supporters to tried one sweet and one sour ice-cream. Findings show that sweetness was 
promoted in positive situations (after a win) and depressed in negative ones (following a loss), whilst the reverse was true for sourness. The study design used controlled samples of real food, rather than tastants. Hinting at the way an edible interface could function, and highlighting the value of taste-emotion mapping for designing experiences in-the-wild [27]. A limitation of this study is that the taste was delivered as an addition to the experience of the match instead of exploring how taste can be embedded in the total experience.

Within HCI, studies exploring taste as a design variable have started to emerge. Obrist et al. [22] used a human-centered approach to assess the qualities of basic tastes. They conducted interviews using the Sensory Evaluation Instrument [13] and the Explication Interview Technique [15] to investigate the affective, temporal and embodied characteristics of taste experiences.

Embodiment has also been explored in HCI, particularly in affective mappings for multimodal interfaces. Antle et al. used experts [1] and users [2] to derive mappings for bodily movements. Within studies on taste, Eskine et al. [8] and Chen and Chang [6] both explore how embodiment shapes the mapping between bitter tastes and disgust.

The correlation between emotion and taste has also been associated with both action and personality by Meier et al. [18]. They found that preference for sweet foods could be used as a predictor of prosocial personalities, intentions and actions.

The potential for taste to influence mood and intention suggests it could form a powerful tool for affective, interpersonal communication. In particular, the influence of embodiment on the interaction between food and affective response suggests a novel direction for tangible interfaces through which physical, bodily experience on the one hand, and affective, cognitive experience on the other hand can be simultaneously stimulated. This study aims to lay the foundations for the application of taste and emotion in the development of affective, edible interfaces.

\section{METHOD}

To understand how taste-emotion mappings are perceived, created and used in an applied context, we conducted semistructured interviews lasting between 30 and 45 minutes with food industry professionals. The interviews focused on experts' emotions; both discrete [7] and continuous ones [29]. The study consisted of three parts. First, we asked participants to place a typical food experience of each of the five basic tastes, i.e. sweet, sour, salt, bitter and umami, onto Russell's circumplex map [29]. Second, we asked them to describe emotions they associated with each of the basic tastes. Third, we asked for tastes or flavors they associated with Ekman's discrete emotions [7]. As we wished to explore how tastes' intensity influenced the mappings we did not suggest any levels, allowing participants to define themselves how different intensity levels influenced their affective responses.

Interviews took place in person or via the phone and were recorded and fully transcribed. The data analysis involved a hybrid approach where existing concepts were used for the deductive coding while new concepts grounded on the empirical data, contributed to inductive coding [10]. The deductive coding included the mapping of sweet to positive valence, and bitterness, saltiness and sourness to negative valence [3,26,28]. The resulting coding list was iteratively refined in the light of the interview data, as new codes emerged under the themes of intensity of taste, the typical foodstuffs triggering taste, typical flavors stimulating multiple tastes, the influence of narrative on taste and its embodied response. The coding was conducted by the primary author and iterated through weekly discussion over several months between authors until stability was achieved.

\subsection{Participants}

We recruited 7 food industry professionals with knowledge of how tastes can be used to create experiences ( 3 female, 4 male) aged between 29 and $38(M=32.9, \mathrm{SD}=2.7)$. The convenience sample consists of chefs $(n=4)$ and food designers $(n=3)$. Participants were recruited all work within the UK.

\section{FINDINGS}

We now report the findings of our mapping exercises starting with the circumplex model followed by the identified tasteemotion mappings.

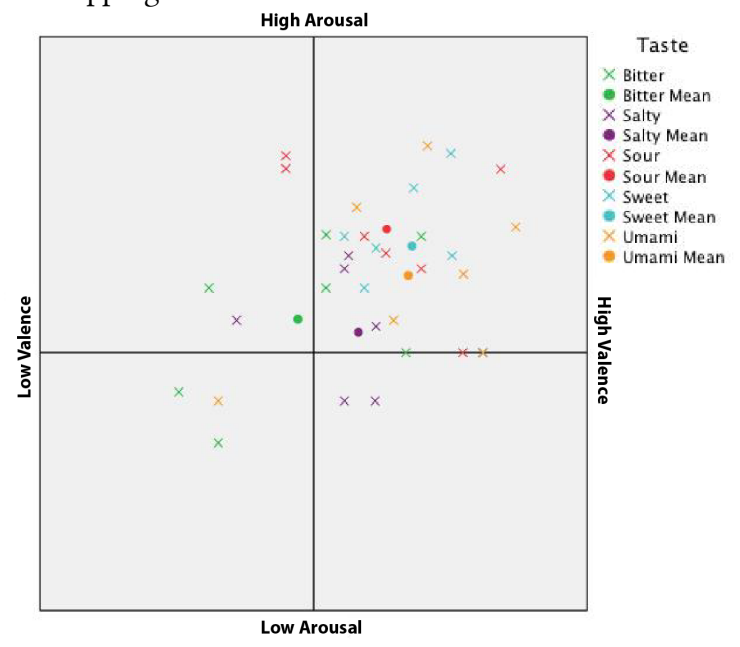

Figure 1 : Circumplex Mapping of Tastes on Emotional Arousal and Valence

Figure 1 shows each participant's mapping of tastes on the circumplex model. A few trends are emerging from these findings. First, most tastes appear in the high arousal-high valence top right quadrant. The highest emotional arousal is triggered by sour taste, whilst the highest valence by sweet and umami tastes. Salty and bitter are both perceived as lower arousal, with bitter being the only taste with an average negative valence.

\subsection{Tastes with Positive Affective Response}

The association between the sweet taste and positive affective response was highlighted by our participants, often 
with the additional association of comfort $(n=6)$. Intriguingly, for happiness, a combination of sweet, umami and salty tastes was mentioned. These tastes were described as 'like mother's milk kind of thing. You know it is an innately pleasurable trio' [P5]. This suggests how taste can still play an important role in affective response even at the level of a complex, compound experience. Another important finding is the mapping of umami to happiness $(\mathrm{n}=3)$ and positive affective response. This contrasts with less conclusive prior findings on the mapping between umami and discrete emotions [14,37,39].

\subsection{Narrative and Memory}

This section outlines the value of narrative and episodic memories in food experience. Where reported, the positive affect of both bitter and sour tastes was linked to narratives reflecting childhood memories. Enjoyment of bitter tastes is known to be a learnt response [4] and our findings confirm this process of acquiring a taste for bitterness: 'there is a sort of desire to attain that level of maturity with bitterness' [P1].

Sour sweet treats were a common example of a food stuff in which the sour taste was enjoyed. Often this response was linked to early childhood memories: 'it reminds me of sour sweeties like really sour stuff, reminds me of childhood' [P5]. For bitter and sour, respondents often pointed to prototypical food stuffs, such as sour sweets or coffee, that were enjoyed.

Sourness was most strongly related to surprise $(n=7)$ and excitement $(n=3)$, which supports the circumplex map findings in which sour had the highest mean arousal. One interviewee described sourness as 'adventure [...] a sort of bravery, I think that is the emotional bravery and a desire to enjoy something that actually is quite difficult to enjoy' [P1]. With both bitter and sour tastes, ideas of transgression, bravery and maturity also emerge.

\subsection{Tastes with Negative Affective Response}

Bitterness was also mapped to anger, fear and disgust, confirming previous lab-based findings $[6,9,12]$. Fear however was interpreted in a more physical manner: 'I feel caffeine just bunches up your energy really tightly and uses it all up at once and leaves you completely useless afterwards' [P6]. Sour provided little evidence of negative affect, although interviewees reported 'some sort of displeasure [which makes it] exciting' [P5].

\subsection{Salty Taste}

A surprising finding was that salty taste was mapped to neither positive nor negative affective response. In the circumplex model, we can see that salty taste is associated with both a lower arousal and valence than other tastes. Our interviewees reported this as 'some kind of contemplation' [P3], 'idling' [P4] and 'mellow' [P2].

\subsection{Non-Taste Food Characteristics}

No taste mappings were reported by several participants for both anger and sadness. For anger; chili, overcooked and burnt foodstuffs were proposed. Anger-inducing foods were often defined by their textures, being 'quite sharp and if you try to eat [them, they] will scratch your gums up a bit' [P6]. The same participant also described the temporal aspect of 'anger' foods as 'quite hard to escape from [as they have] rolling, ongoing consequences and need time to calm down, which seems perfect for [anger].' Sadness was described through a lack of food, or bland and thin foods; not a taste mapping but highlighting how the absence of a taste could be manipulated in experience design.

\subsection{Taste Intensity}

Herbert et al. [12] argued that the intensity of taste is positively correlated to emotional response. Our findings suggest a more nuanced understanding, such as an inverse $U$ curve, where emotional response is positive for most levels of intensity but becomes negative for the lowest intensity level (i.e. bland foodstuff) and the highest. For example, too much sweetness was described as negative, 'there is definitely an upper limit to what you can consume because it will very quickly start to make you sick, so it is sort both an attractive thing but also something that you're aware can turn on you a bit' [P6]. Here the physical consequence informs the wariness of very sweet tasting foods.

\section{DISCUSSION}

We now discuss the key findings in relation to prior work and how they contribute to the study's research questions. With respect to circumplex model, our findings confirmed the previous lab-based ones [3,14,23,28,39] on sweet-positive and bitter-negative mappings. However, we found intensity to not be simply correlated with affective response, and found new mappings for sour and umami tastes to arousing and positive affective reactions.

While prior lab-based findings suggested a positive correlation between taste intensity and strength of affective response [12] our findings indicate that this applies only for the middle levels of taste intensity, but intensities that are too low or too high trigger a negative response. These findings are also supported by Yamaguchi and Takahashi's [39] study on the taste intensity of foodstuffs.

Physical response has been previously mentioned in relation to tastes supporting the idea of an embodied approach to mappings $[6,8]$. Our findings confirm this, with caffeine being identified to trigger fear as a result of digesting the compound. Similarly, the sour-surprise mapping is informed by the 'tingly and exciting' physical sensation of the taste. This suggests that when designing experiences with tastes it is important to pay attention to the longer term affects as well as the 'in-themoment' influences of embodiment. For example, the connection between highly sweet tastes and nausea could arise due to a single intense dose or an accumulation of doses over time, both potentially intended to represent or trigger positive responses but in fact entailing negative ones.

For sour and bitter tastes, findings indicate several prototypical natural foodstuffs. Evidence from rat studies has shown that such foodstuffs can generate a similar but more informationally rich neurological stimulation than pure tastants [30]. Prior findings support the claims of the multisensory flavor 
perception [32,33] because of the extra sensory activity experienced from a food compared to a taste. However, it also shows the possibility of using certain foods to create responses akin to those achieved through pure tastants.

Narrative was highlighted as a key method for interviewees to construct experience from taste, offering insight into food designers' means of experience construction. This method was particularly apparent in bitter and sour tastes where the main narrative described crossing the boundary from pleasant to unpleasant. The transgression of enjoying an initially unpleasant sensation opens a new design space for creating experiences of transformation. Salt provides an intriguing counter point to the other tastes by acting as a 'negative space', meaningful in its lack of specific emotional response

Obrist et al. [22] have previously highlighted the variable nature of affective response to taste over time. Temporality is an under explored quality of both food and taste experience. Tuters and Kera [34] offered a framework for understanding temporal possibilities via metabolic interaction, describing how food is involved in a long chain of interactions that form one, complete experience. For designers, this adds a depth to the way taste can be used, through operating on several temporal scales simultaneously.

\section{DESIGN IMPLICATIONS FOR EDIBLE INTERFACES}

We now discuss the implications of our findings for designing user experience in HCI through an increased awareness of ecologically valid taste-emotion mappings. We also discuss how intensity and temporality can be considered, together with the limitations of taste as a medium and its potential contribution of integration in multisensory interactive systems.

\subsection{Awareness of Taste-Emotion Mappings}

Our findings aim to sensitize designers of edible interfaces to the importance of the taste-emotion mappings in naturalistic settings, both across the circumplex and discrete model of emotions. While there are individual differences in people's mappings [24], our findings point towards some novel relationships such as that of sourness and surprise, and high levels of sweetness and negative affect. Findings also confirmed prior mappings for bitter and sweet. The agreement on these mappings in both abstract and naturalistic settings indicates these to be the best starting point for further exploration.

Designers of edible interfaces should also be aware of the nonlinear relationship between taste intensity and the arousal of positive emotions. Our findings open up the possibility of intensity as a variable of the taste experience, manipulated to change the perception of a singular taste from pleasure to nausea (in the case of sweet) or refined maturity to disgust (in the case of bitter).

\subsection{Design for Temporality of Edible Interfaces}

Taste also affords the opportunity for experiences that transform over time. We have seen how narratives and memory can influence this at one level, whilst lingering tastes and digestion can have an impact on another. These compound timelines offer the tools for designers to create complex experiences that last beyond the tasting, connecting back to the user's memory but also creating an on-going experience into the future.

Here we could see the manipulation of the functional properties of compounds that afford taste. Caffeine is a bitter tastant but also a stimulant, sucrose similarly for sweet. At this point taste extends into physical response and embodiment plays a role. A unique quality of edible interfaces is that computing is enabled to extend into the user's body in an ephemeral way, offering designers a bodily space for interaction that doesn't involve permanent or intrusive application of technology.

\subsection{Exploiting the Limitations of Taste- Emotion Mappings}

Findings also indicate that specific emotions such as sadness are difficult to be mapped to taste. This opens up the design space of co-creating tastes that people can identify with sadness, possibly leveraging the power of narrative. In an interactive system this could be constructed at a personal or interpersonal level. The development of culturally specific meanings for emojis [16] could provide some interesting insight into such a development. Anger also didn't map to taste, but our findings support the possibility of building on knowledge of multisensory integration [31], using touch or temperature to augment the taste sensation to create the required response.

\section{CONCLUSIONS}

This paper reports on interviews with 7 chefs and food designers to understand the validity of taste-emotion mappings for the design of ecologically valid user experiences. Sweet-positive and bitter-negative mappings were confirmed and a more nuanced understanding of to taste intensity on affective response has emerged. Sour, salty and umami tastes were found to exhibit mappings differing from lab-based findings. Findings have led to three design implications highlighting the value of sensitizing designers about the taste-emotion mapping, the role of temporality, and of opportunities of exploring currently unavailable mappings for sadness and anger.

\subsection{Limitations}

In the interviews, participants were relying on their memory of taste experiences and taste sensitivity as not accounted for. Further work is needed to explore these taste experiences in real time and to explore the influence of sensitivity.

\section{ACKNOWLEDGMENTS}

This work was supported by the Designing Multisensory Interactions: Emotional User Experience of 3D Printed-Food Consumption project funded by the Engineering and Physical Sciences Research Council and Dovetailed Ltd. under the Cooperative Awards in Science and Engineering (CASE). 


\section{REFERENCES}

[1] Alissa N. Antle, Greg Corness, and Milena Droumeva. 2009. What the body knows: Exploring the benefits of embodied metaphors in hybrid physical digital environments. Interacting with Computers 21, 1-2: 66-75. https://doi.org/10.1016/j.intcom.2008.10.005

[2] Saskia Bakker, Alissa N. Antle, and Elise Van Den Hoven. 2012. Embodied Metaphors in Tangible Interaction Design. Personal Ubiquitous Comput. 16, 4: 433-449. https://doi.org/10.1007/s00779-011-0410-4

[3] Wender L. P. Bredie, Hui Shan Grace Tan, and Karin Wendin. 2014. A Comparative Study on Facially Expressed Emotions in Response to Basic Tastes. Chemosensory Perception 7, 1: 1-9. https://doi.org/10.1007/s12078-0149163-6

[4] Paul A.S. Breslin. 2013. An Evolutionary Perspective on Food Review and Human Taste. Current biology: $C B$ 23, 9: R409-R418. https://doi.org/10.1016/j.cub.2013.04.010

[5] Kai Qin Chan, Eddie M. W. Tong, Deborah H. Tan, and Alethea H. Q. Koh. 2013. What do love and jealousy taste like? Emotion 13, 6: 1142-1149. https://doi.org/10.1037/a0033758

[6] Bin-Bin Chen and Lei Chang. 2012. Bitter struggle for survival: Evolved bitterness embodiment of survival motivation. Journal of Experimental Social Psychology 48, 2: 579-582. https://doi.org/10.1016/j.jesp.2011.11.005

[7] Paul Ekman. 1992. An Argument for basic emotions. Cognition and Emotion 6, 3/4: 169-200.

[8] Kendall J. Eskine, Natalie A. Kacinik, and Jesse J. Prinz. 2011. A Bad Taste in the Mouth: Gustatory Disgust Influences Moral Judgment. Psychological Science 22, 3: 295-299. https://doi.org/10.1177/0956797611398497

[9] Kendall J. Eskine, Natalie A. Kacinik, and Gregory D. Webster. 2012. The Bitter Truth about Morality: Virtue, Not Vice, Makes a Bland Beverage Taste Nice. PLoS ONE 7, 7: 1-4. https://doi.org/10.1371/journal.pone.0041159

[10] Jennifer Fereday and Eimear Muir-Cochrane. 2006. Demonstrating rigor using thematic analysis: A hybrid approach of inductive and deductive coding and theme development. International journal of qualitative methods 5, 1: 80-92.

[11] E. Greimel, M. Macht, E. Krumhuber, and H. Ellgring. 2006. Facial and affective reactions to tastes and their modulation by sadness and joy. Physiology and Behavior 89, 2: 261-269. https://doi.org/10.1016/j.physbeh.2006.06.002

[12] Cornelia Herbert, Petra Platte, Julian Wiemer, Michael Macht, and Terry D. Blumenthal. 2014. Supertaster, super reactive: Oral sensitivity for bitter taste modulates emotional approach and avoidance behavior in the affective startle paradigm. Physiology \& Behavior 135: 198-207. https://doi.org/10.1016/j.physbeh.2014.06.002

[13] Katherine Isbister, Kristina Höök, Michael Sharp, and Jarmo Laaksolahti. 2006. The Sensual Evaluation Instrument: Developing an Affective Evaluation Tool. In
Proceedings of the SIGCHI Conference on Human Factors in Computing Systems (CHI '06), 1163-1172. https://doi.org/10.1145/1124772.1124946

[14] Hideaki Kashima and Naoyuki Hayashi. 2011. Basic Taste Stimuli Elicit Unique Responses in Facial Skin Blood Flow. PLOS ONE 6, 12: e28236. https://doi.org/10.1371/journal.pone.0028236

[15] Ann Light. 1999. Vermersch'sExplicitation'Interviewing Technique Used in Analysing Human-Computer Interaction. COGNITIVE SCIENCE RESEARCH PAPERUNIVERSITY OF SUSSEX CSRP. Retrieved January 9, 2017 from http://www.sussex.ac.uk/informatics/cogslib/reports/csrp/cs rp513.ps.Z

[16] Xuan Lu, Wei Ai, Xuanzhe Liu, Qian Li, Ning Wang, Gang Huang, and Qiaozhu Mei. 2016. Learning from the Ubiquitous Language: An Empirical Analysis of Emoji Usage of Smartphone Users. In Proceedings of the 2016 ACM International Joint Conference on Pervasive and Ubiquitous Computing (UbiComp '16), 770-780. https://doi.org/10.1145/2971648.2971724

[17] Michael Macht and Jochen Mueller. 2007. Increased negative emotional responses in PROP supertasters. Physiology \& Behavior 90, 2-3: 466-472. https://doi.org/10.1016/j.physbeh.2006.10.011

[18] Brian P. Meier, Sara K. Moeller, Miles Riemer-Peltz, and Michael D. Robinson. 2012. Sweet taste preferences and experiences predict prosocial inferences, personalities, and behaviors. Journal of Personality and Social Psychology 102, 1: 163-174. https://doi.org/10.1037/a0025253

[19] Masashi Nakagawa, Keiko Mizuma, and Takako Inui. 1996. Changes in taste perception following mental or physical stress. Chemical senses 21, 2: 195-200.

[20] Corinna Noel and Robin Dando. 2015. The effect of emotional state on taste perception. Appetite 95: 89-95. https://doi.org/10.1016/j.appet.2015.06.003

[21] Lauri Nummenmaa, Enrico Glerean, Riitta Hari, and Jari K. Hietanen. 2014. Bodily maps of emotions. Proceedings of the National Academy of Sciences 111, 2: 646-651. https://doi.org/10.1073/pnas.1321664111

[22] Marianna Obrist, Rob Comber, Sriram Subramanian, Betina Piqueras-Fiszman, Carlos Velasco, and Charles Spence. 2014. Temporal, Affective, and Embodied Characteristics of Taste Experiences: A Framework for Design. In Proceedings of the 32Nd Annual ACM Conference on Human Factors in Computing Systems (CHI '14), 2853-2862. https://doi.org/10.1145/2556288.2557007

[23] C. Park, D. Looney, and D. P. Mandic. 2011. Estimating human response to taste using EEG. In 2011 Annual International Conference of the IEEE Engineering in Medicine and Biology Society, 6331-6334. https://doi.org/10.1109/IEMBS.2011.6091563

[24] Danielle R. Reed, Toshiko Tanaka, and Amanda H. McDaniel. 2006. Diverse tastes: Genetics of sweet and 
bitter perception. Physiology \& behavior 88, 3: 215-226. https://doi.org/10.1016/j.physbeh.2006.05.033

[25] Ryan S. Ritter and Jesse Lee Preston. 2011. Gross gods and icky atheism: Disgust responses to rejected religious beliefs. Journal of Experimental Social Psychology 47, 6: 1225-1230. https://doi.org/10.1016/j.jesp.2011.05.006

[26] O Robin, S Rousmans, A Dittmar, and E Vernet-Maury. 2003. Gender influence on emotional responses to primary tastes. Physiology \& Behavior 78, 3: 385-393. https://doi.org/10.1016/S0031-9384(02)00981-2

[27] Yvonne Rogers. 2011. Interaction Design Gone Wild: Striving for Wild Theory. interactions 18, 4: 58-62. https://doi.org/10.1145/1978822.1978834

[28] S. Rousmans, O. Robin, A. Dittmar, and E. Vernet-Maury. 2000. Autonomic Nervous System Responses Associated with Primary Tastes. Chemical Senses 25, 6: 709-718. https://doi.org/10.1093/chemse/25.6.709

[29] James A. Russell. 2003. Core affect and the psychological construction of emotion. Psychological Review 110, 1: 145-172. https://doi.org/10.1037/0033-295X.110.1.145

[30] Joshua D. Sammons, Michael S. Weiss, Jonathan D. Victor, and Patricia M. Di Lorenzo. 2016. Taste coding of complex naturalistic taste stimuli and traditional taste stimuli in the parabrachial pons of the awake, freely licking rat. Journal of Neurophysiology 116, 1: 171-182. https://doi.org/10.1152/jn.01119.2015

[31] Charles Spence. 2003. Crossmodal Attention and Multisensory Integration: Implications for Multimodal Interface Design. In Proceedings of the 5th International Conference on Multimodal Interfaces (ICMI '03), 3-3. https://doi.org/10.1145/958432.958435

[32] Charles Spence. 2010. The multisensory perception of flavour. Psychologist 23, 9: 720-723.
[33] Charles Spence. 2015. Multisensory Flavor Perception. Cell 161, 1: 24-35. https://doi.org/10.1016/j.cell.2015.03.007

[34] Marc Tuters and Denisa Kera. 2014. Hungry for Data: Metabolic Interaction from Farm to Fork to Phenotype. In Eat, Cook, Grow: Mixing Human-Computer Interactions with Human-Food Interactions, Jaz Hee-Jeong Choi, Marcus Foth and Greg Hearn (eds.). The MIT Press, Cambridge, Mass., 243-264.

[35] Qian Janice Wang, Sheila Wang, and Charles Spence. 2016. "Turn Up the Taste": Assessing the Role of Taste Intensity and Emotion in Mediating Crossmodal Correspondences between Basic Tastes and Pitch. Chemical Senses 41, 4: 345-356 https://doi.org/10.1093/chemse/bjw007

[36] J. Wei and A. D. Cheok. 2012. Foodie: play with your food promote interaction and fun with edible interface. IEEE Transactions on Consumer Electronics 58, 2: 178-183. https://doi.org/10.1109/TCE.2012.6227410

[37] Karin Wendin, Bodil H. Allesen-Holm, and Wender L. P. Bredie. 2011. Do facial reactions add new dimensions to measuring sensory responses to basic tastes? Food Quality and Preference 22, 4: 346-354. https://doi.org/10.1016/j.foodqual.2011.01.002

[38] Bruno Wicker, Christian Keysers, Jane Plailly, Jean-Pierre Royet, Vittorio Gallese, and Giacomo Rizzolatti. 2003. Both of Us Disgusted in My Insula: The Common Neural Basis of Seeing and Feeling Disgust. Neuron 40, 3: 655664. https://doi.org/10.1016/S0896-6273(03)00679-2

[39] Shizuko Yamaguchi and Chikahito Takahashi. 1984. Hedonic Functions of Monosodium Glutamate and Four Basic Taste Substances Used at Various Concentration Levels in Single and Complex Systems. Agricultural and Biological Chemistry 48, 4: 1077-1081. https://doi.org/10.1271/bbb1961.48.1077 